\title{
Sources of error in AC measurement of skin conductance
}

\author{
Ørjan G. Martinsen ${ }^{1,2,3}$, Oliver Pabst ${ }^{1}$, Christian Tronstad ${ }^{2}$, Sverre Grimnes ${ }^{1,2}$ \\ 1. Department of Physics, University of Oslo, Norway \\ 2. Department of Clinical and Biomedical Engineering, Oslo University Hospital HF, Oslo, Norway \\ 3.E-mail any correspondence to: o.g.martinsen@fys.uio.no
}

\begin{abstract}
Alternating current methods have the potential to improve the measurement of electrodermal activity. However, there are pitfalls that should be avoided in order to perform these measurements in a correct manner. In this paper, we address issues like the choice of measurement frequency, placement of electrodes and the kind of electrodes used. Ignoring these factors may result in loss of measurement sensitivity or erroneous measurements with artifacts that contain little or no physiological information.
\end{abstract}

Keywords: Electrodermal activity, galvanic skin response, skin conductance, bioimpedance.

\section{Introduction}

Skin conductance measurements have been used in psychophysiology for more than a century. Measurements of galvanic skin response (GSR) (also referred to by the more general term electrodermal activity (EDA)) have been reported as early as the last part of the nineteenth century (see [1] for an overview). Through many decades, the method of using direct current (DC) measurements of skin conductance with a constant applied voltage has dominated the EDA literature [2].

However, there are potential complications with the DC method, such as polarization of the electrodes and the corresponding build-up of a counter e.m.f. Furthermore, the use of an alternating current (AC) method instead would enable a simultaneous measurement of any capacitive EDA and also endosomatic EDA (potential responses) [3]. Hence, $\mathrm{AC}$ methods have been investigated for use in EDA studies (see [4] for an overview) [4,5]. While it seems that AC methods solve problems like polarization of the electrodes and electrolysis of the studied tissue, some pitfalls should be avoided to utilize all the possibilities that this method offers. In this paper, we address some obvious pitfalls to be prevented.

\section{Measurement frequency range}

It is generally known that the measurement depth is dependent on the measurement frequency when macroelectrodes (i.e. much larger than the SC thickness) are used for skin admittance measurements. The higher the frequency, the more similar are the electrical properties of the SC and the viable skin [6], and hence the more are the measurements dominated by deeper, viable skin layers. Martinsen et al. [7] found that the stratum corneum (SC) typically dominates the measurements at low frequencies (below $1 \mathrm{kHz}$ ) and that viable skin dominates at higher frequencies (above $100 \mathrm{kHz}$ ).

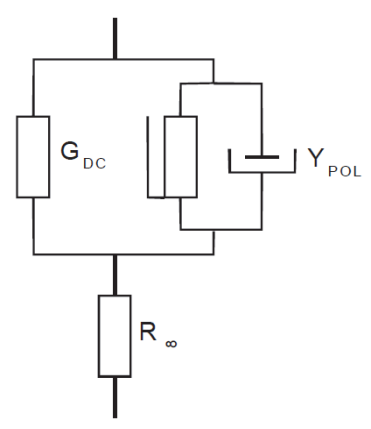

Figure 1: Electrical model of skin

Figure 1 shows a simple equivalent circuit for human skin [8]. The upper part of the model represents the SC and the series resistance $\mathrm{R}_{\infty}$ represents the deeper, viable skin layers. These layers should normally be represented by a more complex electrical model, but at low frequencies, where the SC dominates, a simple resistance is a good approximation. The polarization admittance, $\mathrm{Y}_{\mathrm{POL}}$, represents only the frequency dependent capacitance of the SC and its dielectric loss, while $\mathrm{G}_{\mathrm{DC}}$ represents the conductive properties of both the SC and the sweat ducts $[8,9]$.

To measure the dynamic part of the skin conductance, which is due to sweat activity, it is therefore important to focus the measurements on the $\mathrm{G}_{\mathrm{DC}}$. This is done by keeping the measuring frequency low and by using some kind of synchronous detection to measure only the conductance.

There are two reasons for keeping the measuring frequency low: To avoid the influence of the deeper, viable skin layers, as explained above, and to avoid the influence of the frequency dependent part of the AC conductance. The conductance measured at low frequencies is actually the sum of the DC conductance (free ions in the tissue) and the frequency dependent conductance (dielectric loss in the capacitive properties of the SC).

Figure 2 shows the results of measurements with two Kendall Kittycat ${ }^{\circledR}$ electrodes in the left palm of the hand. A total of 20 measurements were done in different sessions on a healthy, male volunteer, using a Solartron1260/1294 system in a two electrode setup (150 mV rms). 


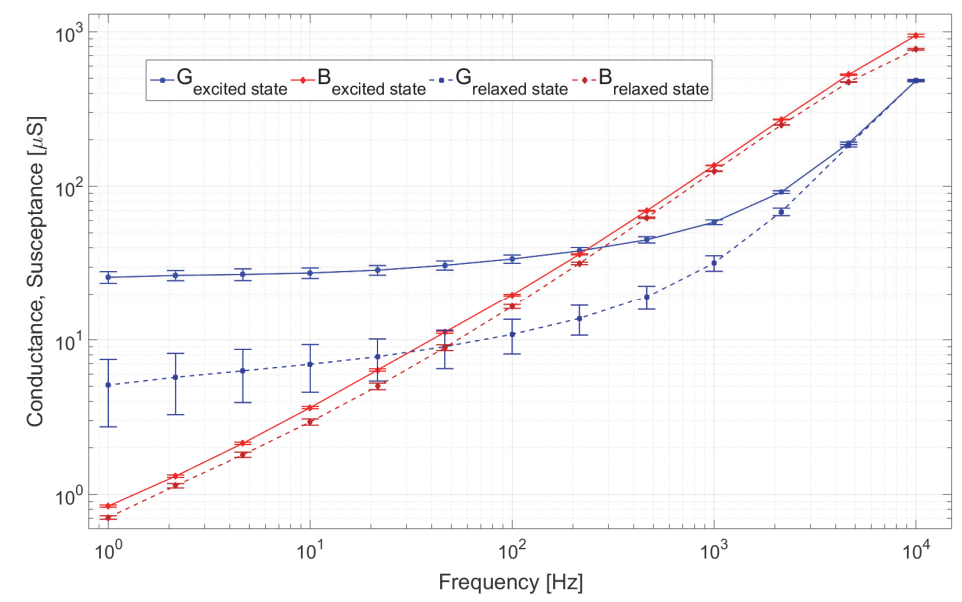

Figure 2: Conductance $\mathrm{G}$ and Susceptance $\mathrm{B}(150 \mathrm{mV} \mathrm{rms})$ as a function of frequency for two palmar Kandall KittyCat ${ }^{\circledR}$ electrodes. The measurements are done for two different levels of sweat activity. Average and standard deviation of 20 measurements for each of the two levels.

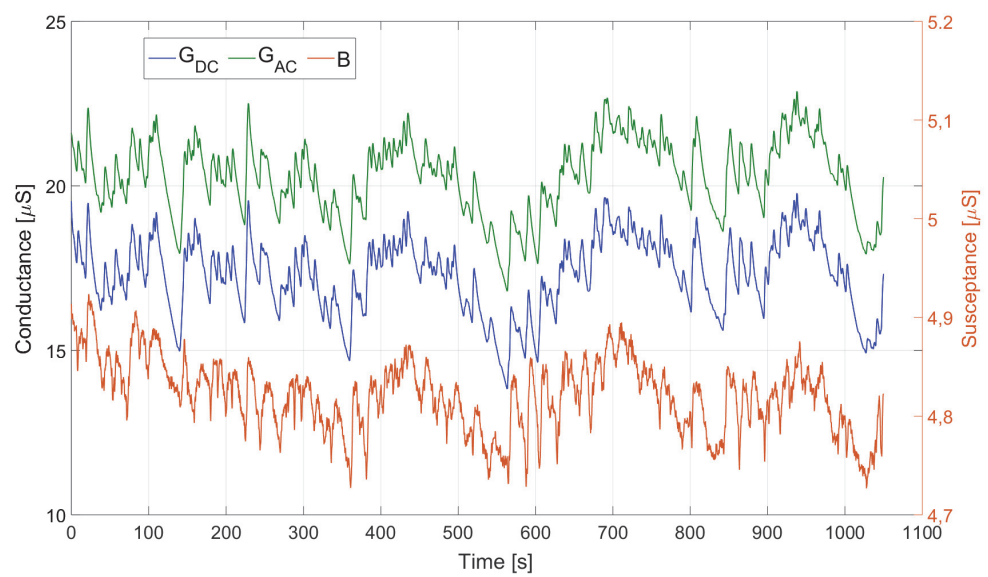

Figure 3: Simultaneous measurement of AC conductance and susceptance ( $20 \mathrm{~Hz}, 500 \mathrm{mV}$ peak amplitude) and $\mathrm{DC}$ conductance $(500 \mathrm{mV})$ on the right palmar skin site during excitation (combing through the test subject's hair). These measurements are done in parallel to the measurements shown in figure 2. It shows the test subject's EDA during the whole session (20 frequency scans).

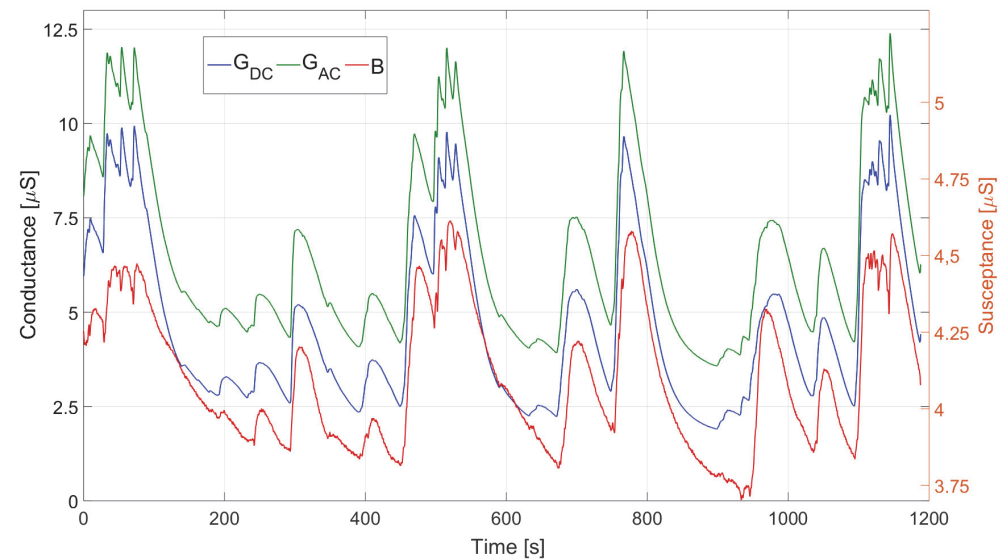

Figure 4: Simultaneous measurement of AC conductance and susceptance ( $20 \mathrm{~Hz}, 500 \mathrm{mV}$ peak amplitude) and DC conductance $(500 \mathrm{mV})$ on the right palmar skin site during relaxation. These measurements are done in parallel to the measurements shown in figure 2. It shows the test subject's EDA during the whole session (20 frequency scans). 
During the sessions, the sweat activity was measured in parallel on the right palmar skin site, which is shown in figures 3 and 4. A custom-made two-electrode based instrument that uses superposition of an AC voltage $(500 \mathrm{mV}$ peak amplitude, $20 \mathrm{~Hz})$ and a $\mathrm{DC}$ voltage $(500 \mathrm{mV})$ to measure AC and DC conductance at the same time was used. During the first measurement session, it was tried to keep the EDA at a high level (figure 3). This was done by combing through the test persons hair. For the seconded session, the test person tried to relax as much as possible. According to figure 2 the conductance measured in the excited state is considerably higher compared with the relaxed state. The difference is mainly due to the sweat activity and therefore the DC conductance.

In the case of excited state, the frequency dependent conductance contributes about $7 \%$ at $20 \mathrm{~Hz}$ and about $31 \%$ at $100 \mathrm{~Hz}$, when assuming the measurement at $1 \mathrm{~Hz}$ is purely due to DC conductance. In the relaxed state, the DC conductance is low and the frequency dependent conductance plays a more significant role even at lower frequencies. The increase from $1 \mathrm{~Hz}$ to $20 \mathrm{~Hz}$ is then about $37 \%$ and from $1 \mathrm{~Hz}$ to $100 \mathrm{~Hz}$ the increase is about $112 \%$. Please note that these results are found for this specific test subject under the described conditions and may be different for other persons and conditions.

Since the frequency dependent part of the conductance has nothing to do with the sweat activity and electrodermal activity (EDR) [10], it is obvious that the measuring frequency for EDA measurements should be kept well below $100 \mathrm{~Hz}$ and preferably as low as possible. The chosen frequency will be a trade-off between the ability of the measuring system to detect quick changes (requires high frequency) and sensitivity for sweat duct activity (requires low frequency), and a frequency in the range $10-20 \mathrm{~Hz}$ may be a good choice in that respect. It should be noted, however, that Nordbotten et al. [11] found that measurements at higher frequencies used for estimation of low-frequency conductance preserves responses, but does not estimate the skin conductance level very well.

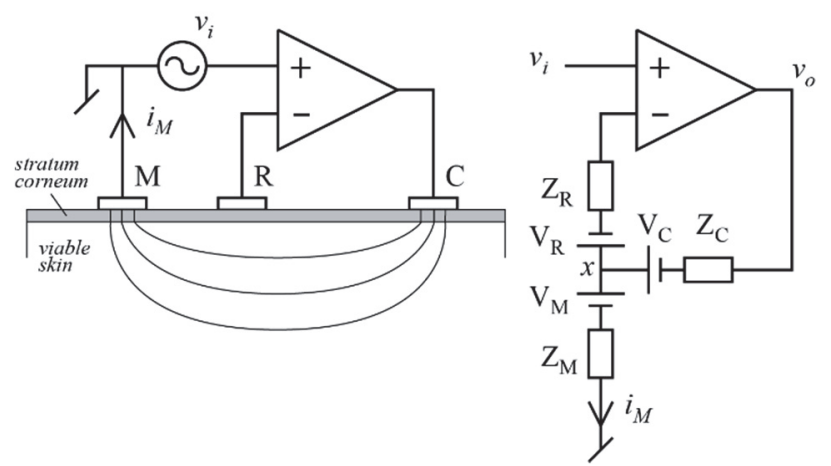

Figure 5: The three-electrode electrical admittance measuring system, showing measuring $(\mathrm{M})$, reference $(\mathrm{R})$ and current carrying (C) electrodes on the skin (left) and with skin impedance and DC potential (right).

\section{Electrode placement}

The three-electrode system enables monopolar measurements of the impedance of the SC beneath one single electrode, without any demands for the size of the other two electrodes [12]. Hence, this electrode system is often used for skin conductance measurements.

The left part of figure 5 shows the three-electrode system with the three electrodes placed on the surface of the skin. For a frequency range where the SC typically dominates the measurements (i.e. typically below $1 \mathrm{kHz}$ ), only the impedance in the SC below the measuring (M) electrode is measured. Because of the feedback through the skin, the operational amplifier will generate the necessary voltage to drive the inverting input to the same voltage as the noninverting input, i.e. $v_{i}$. Since no current flows to the inputs of the (ideal) amplifier, there will be no voltage drop in the SC beneath the reference $(\mathrm{R})$ electrode and since the viable skin can be regarded as equipotential is this case, the voltage applied over the SC beneath the M-electrode will be $v_{i}$. Therefore, if the current $i_{M}$ through the M-electrode is measured (typically by a transresistance amplifier), the impedance of only this part of the SC can be calculated.

The right part of figure 3 shows an equivalent diagram for the measuring set-up, where the impedance of each of the three electrodes are denoted $Z_{M}, Z_{R}$ and $Z_{C}$, respectively. Each of these impedances includes the electrode polarization impedance and the impedance of the epidermal stratum corneum (SC) below the electrode. Compared to the impedance of the SC, the impedance of the living part of the skin is regarded as negligible, which is valid at least at frequencies below $1 \mathrm{kHz}$ for regular macro-electrodes [7]. The skin DC potentials are indicated as batteries in series with these impedances.

Because of the feedback through the skin, the operational amplifier will regulate the output voltage $v_{o}$ so that the voltage at the inverting input is $v_{i}$. Hence, the voltage at the point marked $x$ in the figure will have a voltage

$$
v_{x}=v_{i}+V_{R}
$$

The voltage over $Z_{M}$ will then be

$$
v_{x}-V_{M}=v_{i}+V_{R}-V_{M}=v_{i} \text {, if } V_{R}=V_{M}
$$

It follows from eq. 2 that the voltage over the measured impedance $\mathrm{Z}_{\mathrm{M}}$ is $v_{i}$ only if $\mathrm{V}_{\mathrm{R}}=\mathrm{V}_{\mathrm{M}}$. This means that the Mand R-electrodes must be placed on skin sites with equal, or close to equal, skin potentials. This may not be a problem if $\mathrm{V}_{\mathrm{R}}$ and $\mathrm{V}_{\mathrm{M}}$ are constant DC voltages, since analog measuring systems will typically utilize a high-pass filter to remove any DC offset and digital systems will be immune to any DC offset [13]. However, a transient voltage change, like the ones shown in figure 6 [3], will to a large extent pass the filters and influence the output of both analog and digital lock-in amplifiers. 

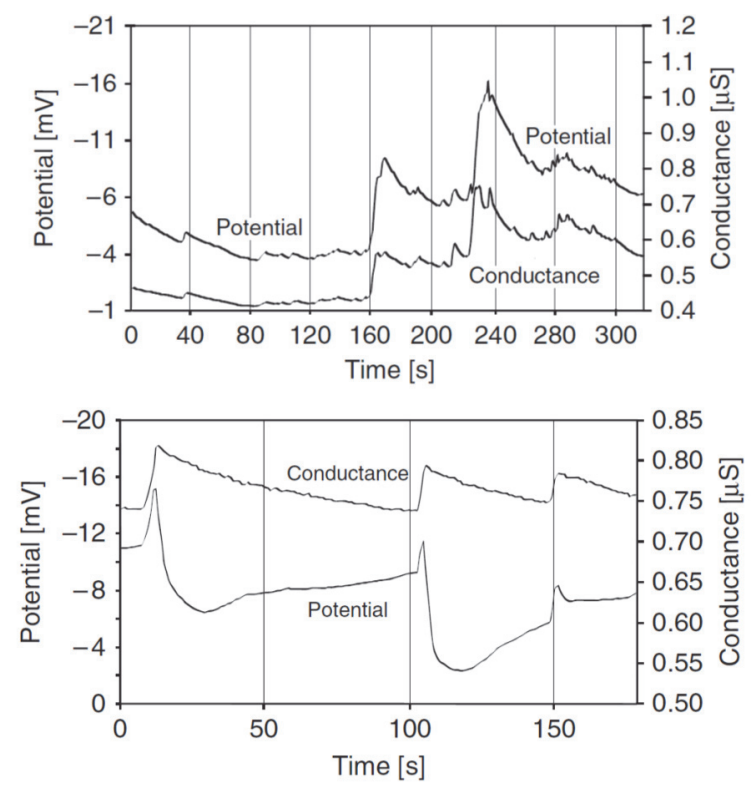

Figure 6: Examples of simultaneously measured skin potential and conductance. From [3] with permission. Top: Highly correlated potential and conductance curves. Bottom: Example of biphasic potential curves.

If, for instance, the M-electrode is placed on a palmar skin site and the R-electrode on the dorsal side of the hand, a sudden increase in the (negative) skin potential inside the hand will lead to a negative response in the measured conductance. Moreover, a decrease in the potential, as we e.g. see in the diphasic signal in figure 6 (bottom), will lead to a positive response in the measured conductance. Hence, the change in skin potential can make the corresponding conductance response appear larger, smaller or even negative. This is one of two possible mechanisms giving rise to false negative conductance responses. The other mechanism is due to using wet gel electrodes and is described in the next section.

\section{Electrode type}

The choice of electrode type is crucial in EDA measurements. A study on electrode gels for skin conductance measurements was done by Tronstad et al. [14]. They found that wet gels would penetrate into the stratum corneum and produce an increasing trend over time in the measured conductance. This is obvious from figure 7 where a Kendall Kittycat $\left.{ }^{(}\right)$solig hydrogel electrode (A) and an Ambu Blue Sensor ${ }^{\circledR}$ Q-00A wet gel electrode (B) were placed on the hypothenar area of the right and left palm of the hands. The increasing trend is clearly visible for the wet gel electrode (B), but not for the solid gel electrode (A). The hatched areas in figures 7 and 8 indicate a period of two minutes, which was initialized by playing a loud sound to the test subject followed by a two-minute physical exercise where the test subject performs squats. Thus, both psychological and thermal sweating was stimulated.
Figure 8 shows corresponding measurements performed on the abdomen of a test subject with the same two electrode types. There is apparently no direct response to the sound stimulus, but the physical activity generates thermal sweating. The two electrode types behave very differently. While the solid gel electrode (A) measures an initial decrease in skin conductance due to the test subject relaxing, the wet gel of electrode B penetrates into the stratum corneum, increasing the baseline level. Apparently, it also penetrates into the sweat ducts, because the conductance response during the physical activity is negative, indicating that the well-conducting gel is pushed out of the sweat ducts and is replaced by sweat of lower conductivity.

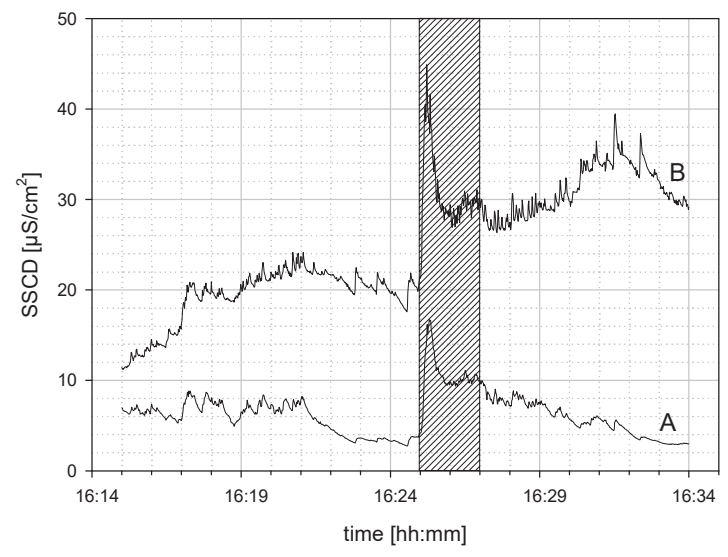

Figure 7: Skin surface conductance density at $22 \mathrm{~Hz}$ measured with Kendall Kittycat ${ }^{\circledR}$ solid hydrogel electrode (A) and Ambu Blue Sensor ${ }^{\circledR}$ Q-00-A wet gel electrode (B) on hypothenar skin sites. From [14]. (C) Institute of Physics and Engineering in Medicine. Reproduced by permission of IOP Publishing. All rights reserved.

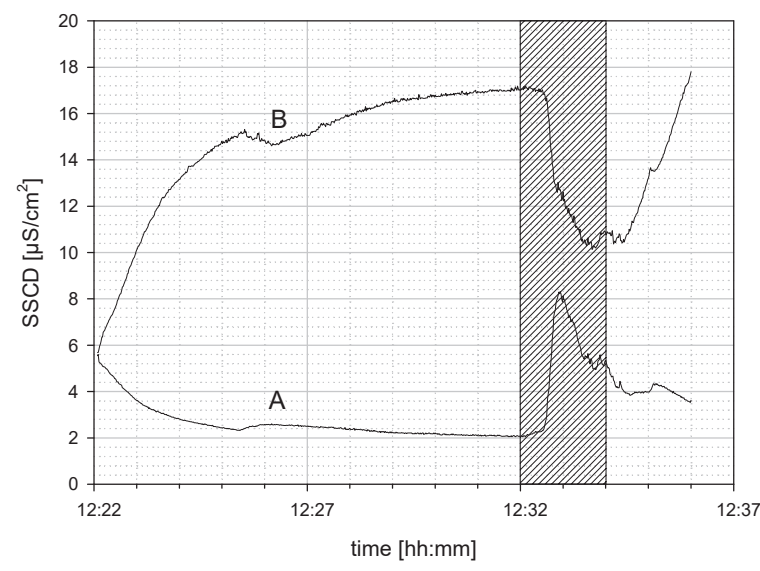

Figure 8: Skin surface conductance density at $22 \mathrm{~Hz}$ measured with Kendall Kittycat ${ }^{\circledR}$ solid hydrogel electrode (A) and Ambu Blue Sensor ${ }^{\circledR}$ Q-00-A wet gel electrode (B) on the abdomen. From [14]. (C) Institute of Physics and Engineering in Medicine. Reproduced by permission of IOP Publishing. All rights reserved.

Although Tronstad et al. [14] speculate that wet gel electrodes may provide higher sensitivity to small responses in partially filled sweat ducts (since the gel can penetrate into the ducts), the general conclusion from this study must be that solid gel electrodes in general are better suited for EDA studies than wet gel electrodes. 


\section{Conclusion}

In this paper, we have argued that AC methods for measurement of skin conductance may have advantages over DC methods in EDA studies, but that there are pitfalls that should be avoided in order to use the AC methods correctly. The correct use of the AC method include a properly chosen measurement frequency, correct use of the electrode system (e.g. a three-electrode system) and the use of electrodes that do not produce significant changes in skin conductance over time.

\section{References}

1. Neumann, E. and R. Blanton, The early history of electrodermal research. Psychophysiology, 1970. 6(4): p. 453475. http://dx.doi.org/10.1111/j.1469-8986.1970.tb01755.x

2. Society for Psychophysiological Research Ad Hoc Committee on Electrodermal Measures, Publication recommendations for electrodermal measurements. Psychophysiology, 2012. 49(8): p. 1017-1034. http://dx.doi.org/10.1111/j.1469-8986.2012.01384.x

3. Grimnes, S., et al., Electrodermal activity by DC potential and AC conductance measured simultaneously at the same skin site. Skin Research and Technology, 2011. 17(1): p. 26-34. http://dx.doi.org/10.1111/j.1600-0846.2010.00459.x

4. Grimnes, S., Psychogalvanic reflex and changes in electrical parameters of dry skin. Medical and Biological Engineering and Computing, 1982. 20(6): p. 734-740. http://dx.doi.org/10.1007/BF02442528

5. Tronstad, C., et al., Electrical measurement of sweat activity. Physiological Measurement, 2008. 29(6): p. S407-S415. http://dx.doi.org/10.1088/0967-3334/29/6/S34

6. Yamamoto, T. and Y. Yamamoto, Electrical properties of the epidermal stratum corneum. Medical and Biological Engineering and Computing, 1976. 14(2): p. 151-158. http://dx.doi.org/10.1007/BF02478741
7. Martinsen, Ø.G., S. Grimnes, and E. Haug, Measuring depth depends on frequency in electrical skin impedance measurements. Skin Research and Technology, 1999. 5(3): p. 179-181. http://dx.doi.org/10.1111/j.1600-0846.1999.tb00128.x

8. Martinsen, Ø.G. and S. Grimnes, On using single frequency electrical measurements for skin hydration assessment. Innovation et Technologie en Biologie et Médecine, 1998. 19(5): p. 395-399.

9. Martinsen, Ø.G., S. Grimnes, and J. Karlsen, Low frequency dielectric dispersion of microporous membranes in electrolyte solution. Journal of Colloid and Interface Science, 1998. 199(2): p. 107-110. http://dx.doi.org/10.1006/jcis.1997.5331

10. Martinsen, Ø.G. and S. Grimnes, Facts and myths about electrical measurement of stratum corneum hydration state. Dermatology, 2001. 202(2): p. 87-89. http://dx.doi.org/10.1159/000051604

11. Nordbotten, B.J., et al., Estimation of skin conductance at low frequencies using measurements at higher frequencies for EDA applications. Physiological Measurement, 2014. 35(6): p. 1011-1018. http://dx.doi.org/10.1088/0967-3334/35/6/1011

12. Grimnes, S., Impedance measurement of individual skin surface electrodes. Medical and Biological Engineering and Computing, 1983. 21(6): p. 750-755. http://dx.doi.org/10.1007/BF02464038

13. Grimnes, S. and Ø.G. Martinsen, Bioimpedance and bioelectricity basics. 3 ed. 2015, London: Academic Press. 471.

14. Tronstad, C., et al., A study on electrode gels for skin conductance measurements. Physiological Measurement, 2010. 31(10): p. 1395-1410. http://dx.doi.org/10.1088/0967-3334/31/10/008 